\title{
Association of UGT2B7, UGT1A9, ABCG2, and IL23R polymorphisms with rejection risk in kidney transplant patients
}

Heloísa Lizotti Cilião, Rossana Batista Oliveira Camargo-Godoy, Marilesia Ferreira de Souza, Mariana Bisarro dos Reis, Lorena lastrenski, Vinicius Daher Alvares Delfino, Silvia Regina Rogatto \& Ilce Mara de Syllos Cólus

To cite this article: Heloísa Lizotti Cilião, Rossana Batista Oliveira Camargo-Godoy, Marilesia Ferreira de Souza, Mariana Bisarro dos Reis, Lorena lastrenski, Vinicius Daher Alvares Delfino, Silvia Regina Rogatto \& Ilce Mara de Syllos Cólus (2017) Association of UGT2B7, UGT1A9, ABCG2, and IL23R polymorphisms with rejection risk in kidney transplant patients, Journal of Toxicology and Environmental Health, Part A, 80:13-15, 661-671, DOI: 10.1080/15287394.2017.1286922

To link to this article: https://doi.org/10.1080/15287394.2017.1286922

\section{Published online: 19 May 2017.}

\section{Submit your article to this journal ¿}

Џ Article views: 101

View Crossmark data ¿

Citing articles: 3 View citing articles $\square$ 


\title{
Association of UGT2B7, UGT1A9, ABCG2, and IL23R polymorphisms with rejection risk in kidney transplant patients
}

\author{
Heloísa Lizotti Ciliãoa, Rossana Batista Oliveira Camargo-Godoyb, Marilesia Ferreira de Souza ${ }^{a}$,

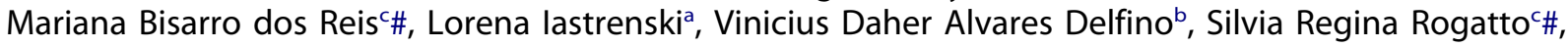 \\ and Ilce Mara de Syllos Cólus ${ }^{\mathrm{a}}$
}

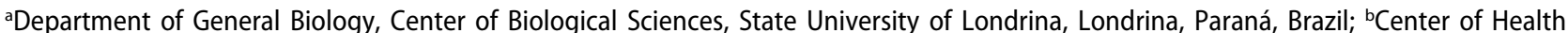
Sciences, State University of Londrina, Londrina, Paraná, Brazil; 'Faculty of Medicine, São Paulo State University (UNESP), Botucatu, São Paulo, Brazil
\end{abstract}

\begin{abstract}
Despite advances in testing compatibility between donor and recipient, graft rejection remains a current concern. Single-nucleotide polymorphisms (SNPs) that codify altered enzymes of metabolism, drug transport, and the immune system may contribute to graft rejection in transplant patients. This study examined the association between SNPs present in genes of these processes and occurrence of graft rejection episodes in 246 kidney transplant patients, 35\% of which were diagnosed with rejection. Genotype-gene expression associations were also assessed. Peripheral blood samples were used for genotyping of 24 SNPs on the following genes: CYP3A4, CYP3A5, CYP2E1, POR, UGT2B7, UGT1A9, ABCB1, ABCC2, ABCG2, SLCO1B1, TNF, IL2, IRF5, TGFB1, NFKBIA, IL10, IL23R, NFAT, and CCR5 by real-time PCR. The analysis of gene expression was performed by RTqPCR. The association between graft rejection episodes and polymorphic variants was assessed using odds ratios. Polymorphisms rs7662029 (UGT2B7) and rs6714486 (UGT1A9) were associated with occurrence of graft rejection episodes, rs7662029 (UGT2B7) exhibited a protective effect (1.85-fold), and rs6714486 (UGT1A9) an increased 1.6-fold increased risk of graft rejection. Among drug transporter genes, only rs2231142 (ABCG2) demonstrated an association with a 1.92-fold decrease in the risk of graft rejection. The immunological SNP rs10889677 (IL23R) was associated with a 1.9-fold enhanced risk of graft rejection. Association between genotypes and gene expression was not detected. Therefore, SNPS of UGT2B7, UGT1A9, ABCG2, and IL23R genes may be useful as candidate markers for screening of risk graft rejection in renal transplant patients. These markers may improve medical decisions, avoiding adverse effects.
\end{abstract}

\section{Introduction}

In the absence of contraindications, kidney transplantation is considered the best option for restoring renal function in patients with advanced chronic kidney disease, not only for medical, social, and economic perspective, but also to increase survival of these patients (Wolfe et al., 1999). Following transplantation procedures, patients use immunosuppressive drugs to reduce the risk of organ rejection such as the triple immunosuppressive regimen consisting of corticosteroids, tacrolimus, and mycophenolate mofetil (MMF). However, prolonged use of these drugs triggers adverse effects (Chapman et al., 2005; Burckart \& Amur, 2010), which limit the long-term benefits of transplantation. The adverse effects influence patients differently owing to inter-individual variability in the response to immunosuppressive drugs, a consequence that may arise, among other factors, from the presence of single-nucleotide polymorphisms (SNPs) in genes involved in the pharmacokinetics/pharmacodynamics of these drugs. These SNPs may be associated with rapid drug clearance, resulting in low plasma chemical concentrations, which initiate rejection episodes (Elens et al., 2011). In contrast, high concentrations of immunosuppressive drugs over a long period after transplantation might lead to (i) decline in renal function and/or graft loss due to nephrotoxicity (Fadili et al., 2013); (ii) loss of renal function due to immunological

CONTACT Ilce Mara de Syllos Cólus ilcecolus@gmail.com Departamento de Biologia Geral, Centro de Ciências Biológicas, Universidade Estadual de Londrina. Rodovia Celso Garcia Cid, Km 380, CEP: 86057-970, Londrina, Paraná, Brasil.

\# Current address: Department of Clinical Genetics, Vejle Hospital, DK and University of Southern Denmark, Denmark.

(๑) 2017 Taylor \& Francis 
damage as evidenced by inflammation and antibodymediated injuries (Ong \& Gaston, 2015), and (iii) development of neoplasia and inflammation (Apel et al., 2013).

Phase I metabolism genes, namely cytochrome P450 (CYPs) and drug-transporting P-glycoprotein genes, such as P-glycoprotein 1 also known as multidrug resistance protein 1 (MDR1, ABCB1 gene), have been reported as the most important genes involved in the pharmacokinetics/pharmacodynamics of calcineurin inhibitors (Hebert, 1997; MacPhee et al., 2005) and the immunosuppressant sirolimus (Sattler et al., 1992). Proteins encoded by UGT2B7 and UGT1A9 phase II metabolism genes are involved in mycophenolate mofetil (MMF) metabolism (Picard et al., 2004), and genes coding for efflux pumps, such as multidrug resistance protein 2 ( $A B C C 2)$, breast cancer resistance protein (BCRP, ABCG2 gene), and organic anion-transporting polypeptide (SLCO1B1), are responsible for the elimination of the major metabolites of MMF (Miura et al., 2008). Therefore, the presence of polymorphisms in these genes might affect the availability of the immunosuppressive drugs and consequently result in a poor correlation between dose, plasma concentration, and therapeutic response (Hesselink et al., 2003; Zununi Vahed et al., 2015).

Polymorphisms in genes of the immune system may also influence the occurrence of graft rejection episodes (Karimi et al., 2014) including cytokines and/or their receptors (such as IL2, IL10, TNF, IL23R, and CCR5), growth factors (TGFB1) and regulatory factors (IRF5). Therefore, SNPs may prove to be of therapeutic use as biomarkers to identify appropriate doses, predict patient tolerance to treatment and risk of graft rejection (Burckart \& Amur, 2010), and consequently have potential to be utilized for prognosis and/or diagnosis of graft rejection episodes.

Taking this into consideration, the aim of this study was to examine the association between incidence of graft rejection and the presence of SNPs in phase I (CYP3A4, CYP3A5, CYP2E1, and $P O R$ ) and phase II (UGT2B7, UGT1A9) metabolism, drug-transporting $(A B C B 1, A B C C 2, A B C G 2$, and SLCO1B1), and immune system genes (TNF, IL2, IRF5, TGFB1, NFKBIA, IL10, IL23R, NFAT, and CCR5).

\section{Methods}

\section{Population studied}

A total of 246 renal transplant patients undergoing post-transplant treatment at the Kidney Institute of Londrina (Londrina, PR, Brazil) were selected. These patients were classified into two groups, those who had graft rejection episodes $(\mathrm{N}=86)$ and those with none $(\mathrm{N}=160)$. Graft rejection episodes were confirmed by histological examination of the graft material obtained through biopsy.

The research protocol was approved by the Ethics Committee for Research in Human Beings at the State University of Londrina (CEP/UEL 153/2013 CAAE: 18263413.4.0000.5231). Patients signed a Free and Informed Consent Form, filled a questionnaire regarding lifestyle and history of environmental exposure, and each individual received a code. Patients were considered smokers and/or alcoholics when they in an interview responded to consuming any amount of cigarettes or alcohol. Patients were divided into two groups: descendant of Caucasian and descendant of Afro/Asian (African $(\mathrm{N}=47)$ and Asian $(\mathrm{N}=2)$ descendant). Information regarding the transplantation process, such as immunosuppressive therapy, graft rejection history, and diseases frequently developed after transplantation, was obtained from medical records of the patients. Peripheral blood samples $(4 \mathrm{ml})$ were collected intravenously from each patient in vacuum blood collection tubes (EDTA 6\%) (Labor Import, Osasco, Brazil).

\section{Analysis of polymorphic allelic variants}

Genomic DNA was extracted from $200 \mu$ l blood, using the mini spin extraction kit (KASVI, Curitiba, Brazil; code K9-0250), following the manufacturer's recommendations. DNA samples were quantified using a NanoDrop 2000 spectrophotometer (ThermoScientific, Waltman, MA, USA).

The genes were selected based upon criteria previously associated with pharmacokinetics of immunosuppressive drugs (metabolism and drug transport genes) or with the immune response (Table 1). Genotyping was performed by real-time PCR in a Quantica thermocycler (TECHNE, Staffordshire, UK), using TaqMan $^{\circledR}$ SNP Genotyping Assays (Applied Biosystems, Foster City, CA, USA), TaqMan Genotyping Master Mix (Applied 
Table 1. Genes Evaluated in Renal Transplant Patients, Their Polymorphisms, and Type of Mutation That Originated Them.

\begin{tabular}{|c|c|c|c|c|}
\hline Gene & SNP_ID & Alternative nomenclature & Change & Chromosome location \\
\hline \multirow[t]{2}{*}{ CYP3A4 } & rs35599367 & $15389 \mathrm{C}>\mathrm{T}(\mathrm{CYP} 3 \mathrm{~A} 4 * 22)$ & Intron & $7 q 22.1$ \\
\hline & rs4646437 & - & Intron & \\
\hline \multirow[t]{2}{*}{ СYРЗА5 } & rs776746 & $6986 \mathrm{~A}>\mathrm{G}\left(\mathrm{CYP} 3 \mathrm{~A} 5^{*} 3\right)$ & Intron, splice acceptor variant & $7 q 21.1$ \\
\hline & rs4646450 & - & Intron & \\
\hline CYP2E1 & rs3813867 & $-1295 G>C(C Y P 2 E 1 * 5 B)$ & Promoter & $10 q 24.3$ \\
\hline POR & rs1057868 & $\left(\mathrm{POR}^{*} 28\right)$ & Exon (Ala503Val) & $7 q 11.2$ \\
\hline UGT1A9 & rs6714486 & $-275 \mathrm{~T}>\mathrm{A}$ & Promoter & $2 q 37$ \\
\hline \multirow[t]{2}{*}{ UGT2B7 } & rs7662029 & $-327 G>A$ & Promoter & $4 q 13$ \\
\hline & rs7438135 & $-900 A>G /-842 A>G$ & Promoter & \\
\hline$A B C B 1$ & rs1045642 & $3435 \mathrm{C}>\mathrm{T}$ & Exon (Ile1145Ile) & $7 q 21.12$ \\
\hline \multirow[t]{2}{*}{$A B C C 2 / M R P 2$} & rs717620 & $-24 \mathrm{C}>\mathrm{T}$ & Promoter ( $\left.5^{\prime} \mathrm{UTR}\right)$ & $10 q 24$ \\
\hline & rs2273697 & $1249 \mathrm{G}>\mathrm{A}$ & Exon (lle417Val) & \\
\hline$A B C G 2 / B C R P$ & rs2231142 & $421 C>A$ & Exon (Lys141Gln) & $4 q 22$ \\
\hline SLCO1B1 & rs4149056 & $521 \mathrm{~T}>\mathrm{C}\left(\mathrm{SLCO} 1 \mathrm{~B} 1{ }^{*} 5\right)$ & Exon (Val174Ala) & $12 p$ \\
\hline$T N F$ & rs1800629 & $-308 \mathrm{G}>\mathrm{A}$ & Promoter & $6 p 21.3$ \\
\hline \multirow[t]{2}{*}{ TGFB1 } & rs 1800470 & $29 \mathrm{~T}>\mathrm{C}$ & Exon (Leu10Pro) & $19 q 13.1-13.3$ \\
\hline & rs1800471 & $915 G>C$ & Exon (Arg25Pro) & \\
\hline IL2 & rs2069762 & $-330 \mathrm{G}>\mathrm{T}$ & Promoter & $4 q 26-q 27$ \\
\hline IL10 & rs1800872 & $-592 C>A$ & Promoter ( $5^{\prime}$ UTR) & $1 q 31-q 32$ \\
\hline IL23R & rs10889677 & - & $3^{\prime}$ UTR & $1 p 31.3$ \\
\hline IRF5 & rs3757385 & - & Promoter & $7 q 32$ \\
\hline NFAT & rs10141896 & NFATC4 & Intron & $14 q 11.2$ \\
\hline NFKBIA & rs696 & $2758 A>G$ & 3'UTR & $14 q 13$ \\
\hline CCR5 & rs333 & CCR5 $\Delta 32$ & Intron deletion & $3 p 21$ \\
\hline
\end{tabular}

Biosystems). Genotyping of the polymorphism rs333 of the CCR5 gene was performed by conventional polymerase chain reaction (PCR) in a Veriti 96-well thermocycler (Life Technologies of Brazil Ltda., São Paulo, Brazil), using $1.5 \mathrm{mM}$ deoxynucleotide (dNTP), $30 \mathrm{mM} \mathrm{MgCl}, 2.5 \mu \mathrm{M}$ of each primer, 0.5 $\mathrm{U}$ Taq DNA polymerase in 10X PCR buffer (Invitrogen-Life Technologies, São Paulo, Brazil), and $20 \mathrm{ng}$ genomic DNA, in a final volume of $15 \mu \mathrm{l}$. The primers used were as follows: sense 5'-ACC AGA TCT CAA AAA GAA-3' and antisense 5'-CAT GAT GGT GAA GAT AAG CCT CA-3'; the PCR conditions were as follows: $94^{\circ} \mathrm{C}$ for $5 \mathrm{~min}, 30$ cycles consisting of $94^{\circ} \mathrm{C}$ for $30 \mathrm{sec}, 57^{\circ} \mathrm{C}$ for $30 \mathrm{sec}$, and $72^{\circ} \mathrm{C}$ for $30 \mathrm{sec}$, followed by a final annealing step of $10 \mathrm{~min}$ at $72^{\circ} \mathrm{C}$. The PCR product had a different size owing to a 32-bp deletion. The fragment was 225-bp long when the prevalent allele was present and $193 \mathrm{bp}$ in the presence of the allele with the deletion. Genotypes were determined by electrophoresis in $10 \%$ polyacrylamide gels stained with silver nitrate (Quimex, Brazil).

\section{Reverse transcription quantitative PCR (RT-qPCR)}

Total RNA from 58 patients was extracted using TRIzol $^{\circledR}$ Reagent (Ambion, Carlsbad, CA, USA) and PureLink ${ }^{\oplus}$ Total RNA Blood Kit (Ambion,
Carlsbad, CA, USA); RNA samples were quantified in a Qubit 2.0 Fluorometer using the Qubit RNA HS Assay (Life Technologies, ref Q32855, Eugene, OR, USA), and its integrity was assessed using the Agilent 2100 Bioanalyzer RNA 6000 LabChip Kit (Agilent Technologies, Inc., Wilmington, DE, USA). For each sample, cDNA was synthesized using $500 \mathrm{ng}$ total RNA and SuperScript ${ }^{\circledR}$ III (Applied Biosystems, Foster City, CA, USA), according to the manufacturer's recommendations. Expression levels of UGT2B7, UGT1A9, ABCG2, and IL23R were evaluated by RT-qPCR. Predesigned oligonucleotide primers were purchased from Sigma-Aldrich (KiCqStart ${ }^{\oplus}$ $\mathrm{SYBR}^{\circledR}$ Green primers), and real-time thermocycler model 7900 (Applied Biosystems, USA) was employed. GUSB (fwd: CCTGCGTCCCACCTA GAATC, rev: ATACGGAGCCCCCTTGTCTG) and PUM1 (fwd: CACAGACACCACCTCCTT CC, rev: CCATTCGTGAGTCCTCCCAG) genes were selected as a reference based on geNorm software analysis (http://medgen.ugent.be/ jvde somp /genorm/).

\section{Statistical analysis}

The continuous variables age (years) and graft survival (years), and the categorical variables (gender, 
degree of HLA compatibility, tobacco and alcohol consumption, ancestry, use of immunosuppressive drugs, development of cancer, diabetes and cardiovascular disease after transplantation) were compared in groups of patients according to graft rejection episodes, using the Student's t-test. Univariate logistic regression analysis was performed associating rejection episodes with each of the variables. HardyWeinberg equilibrium and linkage disequilibrium analysis were performed using HAPLOVIEW version 4.1 (Barrett et al., 2005). The haplotypes and their frequencies were determined using the Program PHASE version 2.1 (Stephens and Donnelly, 2003).

Associations between graft rejection episodes, and genotypes and haplotypes were performed using multivariate logistic regression analysis with SPSS version 20 (IBM, Armonk, NY, USA), and results presented as odds ratios (OR) with a 95\% confidence interval (CI). The degree of HLA compatibility and ancestry $(\mathrm{p}<0.2$ in the univariate logistic regression analysis) were included to adjust the multivariate model. The relative expression of each gene was performed using the $\Delta \Delta \mathrm{Ct}$ method (Pfaffl, 2001). Association between transcripts expression levels and different genotypes was performed using the Student's t-test. For all statistical tests, the criterion used for significance was set at $\mathrm{p}<0.05$.

\section{Results}

The main characteristics of the 246 patients included in this study (145 were men and 101 women) are showed in Table 2. Transplant patients displayed a

Table 2. General and Clinical Characteristics of Kidney Transplant Patients (246) Who Developed and Who Did Not Develop Rejection Episodes.

\begin{tabular}{|c|c|c|c|}
\hline & $\begin{array}{c}\text { General } \\
(\mathrm{n}=246)\end{array}$ & With rejection episode(s) $(\mathrm{n}=86)$ & Without rejection episode $(s)(n=160)$ \\
\hline Characteristics of patients & $\mathrm{N}(\%)$ & $\mathrm{N}(\%)$ & $\mathrm{N}(\%)$ \\
\hline \multicolumn{4}{|l|}{ Gender } \\
\hline Male & $145(58.9)$ & $53(61.6)$ & $92(57.5)$ \\
\hline Female & $101(41.1)$ & $33(38.4)$ & $68(42.5)$ \\
\hline \multicolumn{4}{|l|}{$\begin{array}{l}\text { Degree of HLA } \\
\text { compatibility }\end{array}$} \\
\hline Live HLA-identical & $54(22.0)$ & $11(12.8)$ & $43(26.9)^{*}$ \\
\hline Live HLA-haploidentical & $109(44.3)$ & $39(45.3)$ & $70(43.8)$ \\
\hline Deceased unrelated donor & $83(33.7)$ & $36(41.9)$ & $47(29.4)$ \\
\hline \multicolumn{4}{|l|}{ Smoker } \\
\hline Yes & $18(7.30)$ & $5(5.80)$ & $13(8.10)$ \\
\hline No & $228(92.7)$ & $81(94.2)$ & $147(91.9)$ \\
\hline \multicolumn{4}{|l|}{ Alcoholic } \\
\hline Yes & $35(14.2)$ & $13(15.1)$ & $22(13.8)$ \\
\hline No & $211(85.8)$ & $73(84.9)$ & $138(86.2)$ \\
\hline \multicolumn{4}{|l|}{ Ancestry } \\
\hline Caucasian & $197(80.1)$ & $62(72.1)$ & $135(84.4)^{*}$ \\
\hline Afro or Asian descendant & 49 (19.9) & $24(27.9)$ & $25(15.6)$ \\
\hline \multicolumn{4}{|l|}{$\begin{array}{l}\text { Use of } \\
\text { immunosuppressive }\end{array}$} \\
\hline Cyclosporine & $47(19.1)$ & $20(23.3)$ & $27(16.9)$ \\
\hline Tacrolimus & $112(45.5)$ & $35(40.7)$ & $77(48.1)$ \\
\hline Sirolimus & $16(6.50)$ & $6(7.00)$ & $10(6.30)$ \\
\hline$A Z A^{b}$ & $61(24.8)$ & $20(23.3)$ & $41(25.6)$ \\
\hline $\mathrm{MMF}^{\mathrm{c}}$ & $144(58.5)$ & $49(57.0)$ & $95(59.4)$ \\
\hline Steroids & $244(99.2)$ & $86(100)$ & $158(98.8)$ \\
\hline $\begin{array}{l}\text { MMF+Tacrolimus } \\
\text { +Steroids }\end{array}$ & $87(35.4)$ & $24(27.9)$ & $63(39.4)$ \\
\hline \multicolumn{4}{|l|}{ Development of disease } \\
\hline Cancer & $25(10.2)$ & $7(8.10)$ & $18(11.3)$ \\
\hline Diabetes & $53(21.5)$ & $19(22.1)$ & 34 (21.3) \\
\hline Cardiovascular & $29(11.8)$ & $18(20.9)$ & $11(6.90)^{*}$ \\
\hline Warts & $91(37.0)$ & $35(40.7)$ & $56(35.0)$ \\
\hline
\end{tabular}

${ }^{a}$ HLA-Human Leukocyte Antigen, ${ }^{\mathrm{b}}$ AZA-Azathioprine, 'MMF-mycophenolate mofetil; ${ }^{*} p<0.05$ Student's t-test comparing patients with rejection and without rejection. 
mean age of $48.6 \pm 12.6$ years and duration of transplant ranging from six months to 34 years $(10.6 \pm 8.3$ years). The comparison of these variables showed no significant differences in cases with or without graft rejection episodes

Most grafts were obtained from living donors, $22 \%$ from identical living donors, $44.3 \%$ from haploidentical living donors, and 33.7\% from deceased donors. Most patients were Caucasian (80.1\%), followed by $19.9 \%$ Afro or Asian descendant. Eighteen patients $(7.3 \%)$ reported being smokers, and thirtyfive (14.2\%) consumed alcohol.

Triple immunosuppressive regimen (corticosteroids, tacrolimus, and MMF) was reported in 87 patients (35.4\%); only two subjects $(0.80 \%)$ did not use steroids, and MMF was the second immunosuppressant more frequently utilized by individuals (58.5\%). Among the most prevalent diseases presented after transplantation, the development of diabetes $(21.5 \%)$, cardiovascular diseases (11.8\%), and cancer (10.2\%) were predominant (Table 2).

A total of 86 patients (34.9\%) displayed graft rejection episodes. Multivariate regression analysis demonstrated that the degree of human leukocyte antigen (HLA) compatibility and ancestry is risk factors for graft rejection, and therefore, data were adjusted for these variables. Receiving the graft from an HLA-haploidentical living donor or from a deceased donor resulted in a marked greater than 2 -fold increase in graft rejection risk. Afro or Asian descendant patients also were found to show a significant 2-fold rise in organ rejection risk.

All examined SNPs were in Hardy-Weinberg equilibrium. The multivariate logistic regression analysis revealed four SNP UGT2B7 (rs7662029),
UGT1A9 (rs6714486), ABCG2 (rs2231142), and IL23R (rs10889677) with a significant association with rejection episodes (Table 3). Protection against organ rejection episodes was observed among carriers of the rare alleles of either the rs7662029 (UGT2B7) and rs2231142 (ABCG2) SNP. The association of the genotypes $\mathrm{A} / \mathrm{A}$ and $\mathrm{A} / \mathrm{G}$ in the polymorphism rs7662029 represented a significant 1.85-fold higher protective factor, while genotypes $\mathrm{C} / \mathrm{A}$ and $\mathrm{A} / \mathrm{A}$ at SNP rs2231142 resulted in a 1.92-fold marked decrease in rejection risk

The presence of the polymorphisms rs6714486 (UGT1A9) and rs10889677 (IL23R) resulted in significant elevated risk of organ rejection, with the association of genotypes T/A and A/A at SNP rs6714486 showing a 1.6-fold significant rise in risk of rejection, while subjects with genotypes A/A and A/C at SNP rs10889677 of the IL23R gene displayed a 1.9-fold higher risk of developing rejection.

Two UGT2B7 polymorphisms were in linkage disequilibrium $\left(\mathrm{D}^{\prime}=0.99 ; \mathrm{r}^{2}=0.97\right)$. Linkage disequilibrium was also observed between three other SNPs: rs4646450 (CYP3A5) and rs776746 $(C Y P 3 A 5)\left(\mathrm{D}^{\prime}=0.92 ; \mathrm{r}^{2}=0.51\right), \quad \mathrm{rs} 4646450$ $(C Y P 3 A 5)$ and $r s 4646437(C Y P 3 A 4)\left(D^{\prime}=0.90\right.$; $\left.\mathrm{r}^{2}=0.45\right)$, and rs4646437 (CYP3A4) and rs776746 $(C Y P 3 A 5)\left(\mathrm{D}^{\prime}=0.86 ; \mathrm{r}^{2}=0.68\right)$. Using multivariate logistic regression analysis, the presence of these haplotypes did not show marked association with rejection episodes (data not shown).

ABCG2 and IL23R gene expression analysis demonstrated no marked association with the SNPs mapped in these genes (rs2231142 and rs10889677, respectively) (Figure 1). Due to the low number of patients with genotype AA (rs10889677), analysis of association between
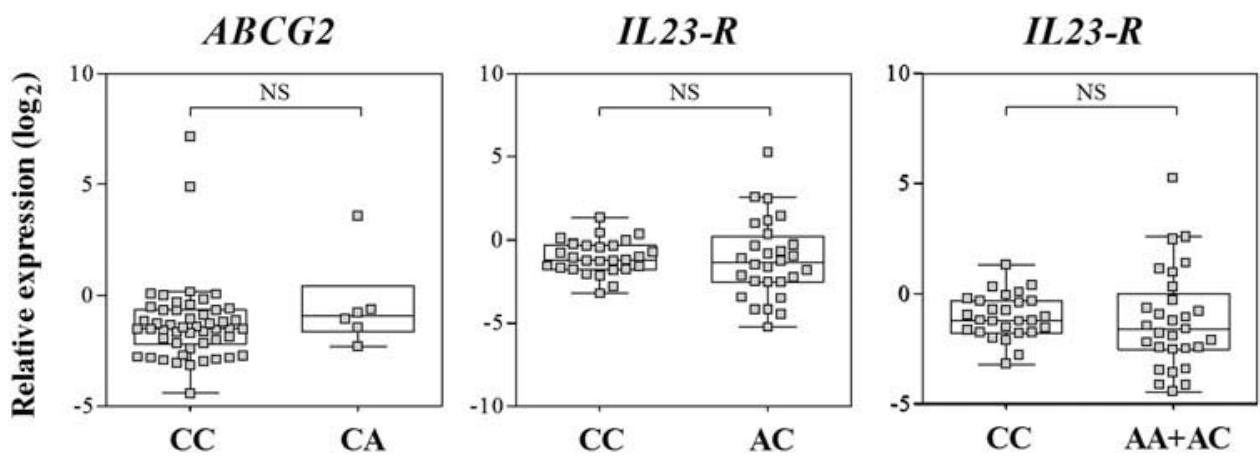

Figure 1. Relative expression of $A B C G 2$ and IL23R detected by RT-qPCR in different genotypes of each gene. The transcript expression values are shown in log scale.*: $P$ value $<0.05$. 
Table 3. Genotypic Frequencies of Genes CYP3A4, CYP3A5, CYP2E1, POR, UGT1A9, UGT2B7, ABCB1, ABCC2, ABCG2, SLCO1B1, TNF, TGFB1, IL2, IL10, IL23R, IRF5, NFAT, NFKBIA and CCR5 in 246 Kidney Transplant Patients, and the Association between Gene Polymorphisms and Rejection Episodes.

\begin{tabular}{|c|c|c|c|c|}
\hline $\begin{array}{l}\text { Genes } \\
\text { (SNP_ID) }\end{array}$ & Genotypes & $\begin{array}{c}\text { With rejection episode(s) } \\
\mathrm{n}(\%)\end{array}$ & $\begin{array}{c}\text { Without rejection episode(s) } \\
\mathrm{n}(\%)\end{array}$ & Odds Ratio ${ }^{\mathrm{a}}$ (C195\%) \\
\hline \multirow{2}{*}{$\begin{array}{l}\text { CYP3A4 } \\
\quad(\text { rs35599367) }\end{array}$} & $\mathrm{C} / \mathrm{C}$ & $81(94.2)$ & $153(95.6)$ & Ref. $^{\mathrm{b}}$ \\
\hline & $\mathrm{C} / \mathrm{T}$ & $5(5.80)$ & $7(4.40)$ & $1.5(0.58-4.0)$ \\
\hline \multirow{4}{*}{$\begin{array}{l}\text { CYP3A4 } \\
\quad(\text { rs4646437) }\end{array}$} & $\mathrm{G} / \mathrm{G}$ & $54(62.8)$ & $106(66.3)$ & Ref. \\
\hline & $\mathrm{G} / \mathrm{A}$ & $25(29.1)$ & $45(28.1)$ & $0.95(0.56-1.6)$ \\
\hline & $\mathrm{A} / \mathrm{A}$ & 7 (8.10) & $9(5.60)$ & $1.1(0.41-3.0)$ \\
\hline & $\mathrm{A} / \mathrm{G}$ and $\mathrm{A} / \mathrm{A}$ & $32(37.2)$ & $54(33.7)$ & $0.98(0.66-1.4)$ \\
\hline \multirow{4}{*}{$\begin{array}{l}\text { CYP3A5 } \\
\quad \text { (rs776746) }\end{array}$} & $\mathrm{G} / \mathrm{G}$ & $53(61.6)$ & $102(63.8)$ & Ref. \\
\hline & $A / G$ & $25(29.1)$ & 49 (30.6) & $0.87(0.47-1.6)$ \\
\hline & $\mathrm{A} / \mathrm{A}$ & $8(9.30)$ & $9(5.60)$ & $1.3(0.41-4.0)$ \\
\hline & $\mathrm{A} / \mathrm{A}$ and $\mathrm{A} / \mathrm{G}$ & $33(38.4)$ & $58(36.2)$ & $0.92(0.57-1.5)$ \\
\hline \multirow{4}{*}{$\begin{array}{l}\text { CYP3A5 } \\
\quad(\text { rs4646450) }\end{array}$} & $\mathrm{C} / \mathrm{C}$ & $33(38.4)$ & $85(53.1)$ & Ref. \\
\hline & $\mathrm{C} / \mathrm{T}$ & $41(47.7)$ & $58(36.2)$ & $1.5(0.78-3.1)$ \\
\hline & $T / T$ & $12(13.9)$ & $17(10.6)$ & $1.6(0.54-4.7)$ \\
\hline & $\mathrm{C} / \mathrm{T}$ and $\mathrm{T} / \mathrm{T}$ & $53(61.6)$ & $75(46.8)$ & $1.6(0.77-3.2)$ \\
\hline \multirow[t]{2}{*}{$\begin{array}{l}\text { CYP2E1 } \\
\quad(\mathrm{rs} 3813867)\end{array}$} & $\mathrm{G} / \mathrm{G}$ & $77(89.5)$ & $136(85.0)$ & Ref. \\
\hline & $\mathrm{G} / \mathrm{C}$ and $\mathrm{C} / \mathrm{C}$ & $9(10.5)$ & $24(15.0)$ & $0.69(0.24-2.0)$ \\
\hline \multirow{4}{*}{$\begin{array}{l}P O R \\
\quad(\text { rs1057868) }\end{array}$} & $\mathrm{C} / \mathrm{C}$ & $42(48.8)$ & $91(56.9)$ & Ref. \\
\hline & $\mathrm{C} / \mathrm{T}$ & $36(41.9)$ & 59 (36.9) & $1.4(0.71-2.7)$ \\
\hline & $\mathrm{T} / \mathrm{T}$ & $8(9.30)$ & $10(6.20)$ & $2.4(0.72-8.4)$ \\
\hline & $\mathrm{C} / \mathrm{T}$ and $\mathrm{T} / \mathrm{T}$ & $44(51.2)$ & $69(43.1)$ & $1.5(0.78-3.0)$ \\
\hline \multirow{2}{*}{$\begin{array}{l}\text { UGT1A9 } \\
\quad \text { (rs6714486) }\end{array}$} & $\mathrm{T} / \mathrm{T}$ & $69(80.2)$ & $142(88.7)$ & Ref. \\
\hline & $\mathrm{T} / \mathrm{A}$ and $\mathrm{A} / \mathrm{A}$ & $17(19.8)$ & 18 (11.3) & $1.6(1.0-2.5)^{*}$ \\
\hline \multirow{4}{*}{$\begin{array}{l}\text { UGT2B7 } \\
\quad \text { (rs7662029) }\end{array}$} & $\mathrm{G} / \mathrm{G}$ & $40(46.5)$ & $48(30.0)$ & Ref. \\
\hline & $A / G$ & 39 (45.4) & 89 (55.6) & $0.57(0.32-1.0)$ \\
\hline & $\mathrm{A} / \mathrm{A}$ & 7 (8.10) & $23(14.4)$ & $0.42(0.16-1.1)$ \\
\hline & $\mathrm{A} / \mathrm{A}$ and $\mathrm{A} / \mathrm{G}$ & $46(53.5)$ & $112(70.0)$ & $0.54(0.3-1.0)^{*}$ \\
\hline \multirow{4}{*}{$\begin{array}{l}\text { UGT2B7 } \\
\quad(\text { rs7438135) }\end{array}$} & $\mathrm{A} / \mathrm{A}$ & $39(45.3)$ & 49 (30.6) & Ref. \\
\hline & $A / G$ & $39(45.3)$ & 87 (54.4) & $0.61(0.35-1.1)$ \\
\hline & $\mathrm{G} / \mathrm{G}$ & $8(9.30)$ & $24(15.0)$ & $0.48(0.19-1.2)$ \\
\hline & $\mathrm{A} / \mathrm{G}$ and $\mathrm{G} / \mathrm{G}$ & $47(54.6)$ & $111(69.4)$ & $0.59(0.33-1.0)$ \\
\hline \multirow{4}{*}{$\begin{array}{l}A B C B 1 \\
\quad(\text { rs 1045642) }\end{array}$} & $\mathrm{C} / \mathrm{C}$ & $34(39.5)$ & $56(35.0)$ & Ref. \\
\hline & $\mathrm{T} / \mathrm{C}$ & $39(45.4)$ & $72(45.0)$ & $0.96(0.61-1.5)$ \\
\hline & $T / T$ & $13(15.1)$ & $32(20.0)$ & $0.70(0.38-1.3)$ \\
\hline & $\mathrm{T} / \mathrm{C}$ and $\mathrm{T} / \mathrm{T}$ & $52(60.5)$ & $104(65.0)$ & $0.88(0.60-1.3)$ \\
\hline \multirow{4}{*}{$\begin{array}{l}A B C C 2 \\
\quad(\text { rs717620) }\end{array}$} & $\mathrm{C} / \mathrm{C}$ & $61(71.0)$ & $104(65.0)$ & Ref. \\
\hline & $\mathrm{C} / \mathrm{T}$ & $23(26.7)$ & 51 (31.9) & $0.82(0.43-1.6)$ \\
\hline & $\mathrm{T} / \mathrm{T}$ & $2(2.30)$ & $5(3.10)$ & $0.65(0.10-4.1)$ \\
\hline & $\mathrm{C} / \mathrm{T}$ and $\mathrm{T} / \mathrm{T}$ & $25(29.0)$ & $56(35.0)$ & $0.81(0.50-1.3)$ \\
\hline \multirow{4}{*}{$\begin{array}{l}A B C C 2 \\
\quad(\mathrm{rs} 2273697)\end{array}$} & $\mathrm{G} / \mathrm{G}$ & $54(62.8)$ & $103(64.4)$ & Ref. \\
\hline & $A / G$ & $30(34.9)$ & $50(31.2)$ & $1.1(0.74-1.7)$ \\
\hline & $\mathrm{A} / \mathrm{A}$ & $2(2.30)$ & $7(4.40)$ & $0.52(1.6-1.7)$ \\
\hline & $\mathrm{A} / \mathrm{G}$ and $\mathrm{A} / \mathrm{A}$ & $32(37.2)$ & 57 (35.6) & $1.0(0.75-1.5)$ \\
\hline \multirow{2}{*}{$\begin{array}{l}A B C G 2 \\
\quad(r s 2231142)\end{array}$} & $\mathrm{C} / \mathrm{C}$ & $76(88.4)$ & $129(80.6)$ & Ref. \\
\hline & $\mathrm{C} / \mathrm{A}$ and $\mathrm{A} / \mathrm{A}$ & $10(11.6)$ & 31 (19.4) & $0.52(0.28-0.96)^{*}$ \\
\hline \multirow{3}{*}{$\begin{array}{l}\text { SLCO1B1 } \\
\quad(\mathrm{rs} 4149056)\end{array}$} & $\mathrm{T} / \mathrm{T}$ & $63(73.2)$ & $107(66.9)$ & Ref. \\
\hline & $\mathrm{T} / \mathrm{C}$ & $20(23.3)$ & $48(30.0)$ & $0.75(0.31-1.8)$ \\
\hline & $\mathrm{C} / \mathrm{C}$ & $3(3.50)$ & $5(3.10)$ & $1.0(0.13-8.2)$ \\
\hline
\end{tabular}


Table 3. (Continued).

\begin{tabular}{|c|c|c|c|c|}
\hline $\begin{array}{l}\text { Genes } \\
\text { (SNP_ID) }\end{array}$ & Genotypes & $\begin{array}{l}\text { With rejection episode(s) } \\
\mathrm{n}(\%)\end{array}$ & $\begin{array}{c}\text { Without rejection episode(s) } \\
\mathrm{n}(\%)\end{array}$ & Odds Ratio ${ }^{\mathrm{a}}$ (C195\%) \\
\hline & $\mathrm{T} / \mathrm{C}$ and $\mathrm{C} / \mathrm{C}$ & $23(26.8)$ & $53(33.1)$ & $0.77(0.40-1.5)$ \\
\hline \multirow{2}{*}{$\begin{array}{l}\text { TNF } \\
\qquad(\mathrm{rs} 1800629)\end{array}$} & $\mathrm{G} / \mathrm{G}$ & $63(73.3)$ & $116(72.5)$ & Ref. \\
\hline & $\mathrm{G} / \mathrm{A}$ and $\mathrm{A} / \mathrm{A}$ & $23(26.7)$ & $44(27.7)$ & $0.94(0.41-2.1)$ \\
\hline \multirow{4}{*}{$\begin{array}{l}\text { TGFB1 } \\
\quad(\text { rs1800470) }\end{array}$} & $\mathrm{C} / \mathrm{C}$ & $26(30.2)$ & $39(24.4)$ & Ref. \\
\hline & $\mathrm{C} / \mathrm{T}$ & 34 (39.6) & $83(51.9)$ & $0.72(0.35-1.5)$ \\
\hline & $\mathrm{T} / \mathrm{T}$ & $26(30.2)$ & $38(23.7)$ & $1.1(0.50-2.5)$ \\
\hline & $\mathrm{C} / \mathrm{T}$ and $\mathrm{T} / \mathrm{T}$ & $60(69.8)$ & $121(75.6)$ & $0.85(0.56-1.3)$ \\
\hline \multirow{2}{*}{$\begin{array}{l}\text { TGFB1 } \\
\quad(\text { rs1800471) }\end{array}$} & $\mathrm{G} / \mathrm{G}$ & $73(84.9)$ & $137(85.6)$ & Ref. \\
\hline & $\mathrm{G} / \mathrm{C}$ and $\mathrm{C} / \mathrm{C}$ & $13(15.1)$ & $23(14.4)$ & $1.1(0.46-2.5)$ \\
\hline \multirow{4}{*}{$\begin{array}{l}\text { IL2 } \\
\quad(r s 2069762)\end{array}$} & $\mathrm{T} / \mathrm{T}$ & $48(55.8)$ & $91(56.9)$ & Ref. \\
\hline & $T / G$ & $32(37.2)$ & $58(36.2)$ & $1.2(0.64-2.1)$ \\
\hline & $\mathrm{G} / \mathrm{G}$ & $6(7.00)$ & $11(6.90)$ & $1.0(0.34-3.2)$ \\
\hline & $\mathrm{T} / \mathrm{G}$ and $\mathrm{G} / \mathrm{G}$ & $38(44.2)$ & $69(43.1)$ & $1.1(0.65-2.0)$ \\
\hline \multirow{4}{*}{$\begin{array}{l}\text { IL } 10 \\
\quad(\mathrm{rs} 1800872)\end{array}$} & $\mathrm{C} / \mathrm{C}$ & $32(37.2)$ & $63(39.4)$ & Ref. \\
\hline & $\mathrm{C} / \mathrm{A}$ & $44(51.2)$ & $77(48.1)$ & $1.1(0.76-1.6)$ \\
\hline & $\mathrm{A} / \mathrm{A}$ & 10 (11.6) & $20(12.5)$ & $0.91(0.51-1.6)$ \\
\hline & $\mathrm{C} / \mathrm{A}$ and $\mathrm{A} / \mathrm{A}$ & $54(62.8)$ & $97(60.6)$ & $1.1(0.81-1.4)$ \\
\hline \multirow{4}{*}{$\begin{array}{l}\text { IL23R } \\
\quad \text { (rs10889677) }\end{array}$} & $\mathrm{C} / \mathrm{C}$ & $33(38.4)$ & $85(53.1)$ & Ref. \\
\hline & $\mathrm{A} / \mathrm{C}$ & $43(50.0)$ & $66(41.3)$ & $1.7(0.98-3.1)$ \\
\hline & $\mathrm{A} / \mathrm{A}$ & $10(11.6)$ & $9(5.60)$ & $3.0(1.1-8.5)^{*}$ \\
\hline & $\mathrm{A} / \mathrm{A}$ and $\mathrm{A} / \mathrm{C}$ & 53 (61.6) & 75 (46.9) & $1.9(1.3-2.7)^{*}$ \\
\hline \multirow{4}{*}{$\begin{array}{l}\text { IRF5 } \\
\qquad(\mathrm{rs} 3757385)\end{array}$} & $\mathrm{T} / \mathrm{T}$ & 17 (19.8) & $29(18.1)$ & Ref. \\
\hline & $\mathrm{T} / \mathrm{G}$ & $43(50.0)$ & $79(49.4)$ & $1.1(0.48-2.6)$ \\
\hline & $\mathrm{G} / \mathrm{G}$ & $26(30.2)$ & $52(32.5)$ & $0.94(0.38-2.3)$ \\
\hline & $\mathrm{T} / \mathrm{G}$ and $\mathrm{G} / \mathrm{G}$ & $69(80.2)$ & $131(81.9)$ & $1.0(0.62-1.8)$ \\
\hline \multirow{2}{*}{$\begin{array}{l}\text { NFAT } \\
\quad(\text { rs10141894) }\end{array}$} & $\mathrm{G} / \mathrm{G}$ & $81(94.2)$ & $150(93.7)$ & Ref. \\
\hline & $\mathrm{G} / \mathrm{T}$ and $\mathrm{T} / \mathrm{T}$ & $5(5.80)$ & $10(6.30)$ & $0.92(0.22-3.8)$ \\
\hline \multirow{4}{*}{$\begin{array}{l}\text { NFKBIA } \\
\quad(\mathrm{rs} 696)\end{array}$} & $\mathrm{G} / \mathrm{G}$ & $30(34.9)$ & $59(36.9)$ & Ref. \\
\hline & $G / A$ & $42(48.8)$ & 73 (45.6) & $0.97(0.41-2.3)$ \\
\hline & $\mathrm{A} / \mathrm{A}$ & $14(16.3)$ & $28(17.5)$ & $0.81(0.26-2.6)$ \\
\hline & $\mathrm{G} / \mathrm{A}$ and $\mathrm{A} / \mathrm{A}$ & $56(65.1)$ & $101(63.1)$ & $0.92(0.63-1.4)$ \\
\hline $\begin{array}{l}\text { CCR5 } \\
\quad(r s 333)\end{array}$ & wt/wt & 77 (91.7) & $150(93.7)$ & Ref. \\
\hline$n=244$ & $w t / \Delta 32$ & $7(8.30)$ & $10(6.30)$ & $1.2(0.48-3.0)$ \\
\hline
\end{tabular}

${ }^{a}$ Odds Ratio (calculated by logistic regression using degree of HLA compatibility and ancestry as covariates);

${ }^{\mathrm{b}}$ Ref - genotype used as reference;

${ }^{*} p<0.05$.

gene expression and genotype was not performed. Furthermore, no detectable transcripts levels were found in peripheral blood samples for UGT1A9 and UGT2B7 genes.

\section{Discussion}

In our cohort of cases, $34.9 \%$ of the patients presented rejection episodes and the average duration of transplants was $10.6 \pm 8.3$ years. This rejection frequency is higher than described by Ro et al. (2012) in subjects where mean duration of kidney transplant of about 4.2 years had an organ rejection rate of $20.5 \%$, and Karimi et al. (2014) reported $28 \%$ rejection in patients after 3 months of transplant. One of the main risk factors for graft dysfunction and rejection is the compatibility between donor and recipient in the genes involved in human leukocyte antigen (HLA). Lack of compatibility reduces the longterm survival of the graft; therefore, molecular typing of HLA improves clinical outcomes 
(Tiercy, 2002). In this study, patients who did not receive a graft from a live donor with identical HLA showed a 2-fold increased risk of graft rejection indicating that HLA compatibility enhancing organ graft survival.

Data also showed that Afro or Asian descendants patients displayed a higher than 2-fold risk of developing rejection than Caucasian descendants patients. This corroborates the findings of Palanisamy et al. (2015), which demonstrated that African-American (AA) patients displayed an 8\% rise in graft loss after 5 years of transplantation compared with non-AA patients. Palanisamy et al. (2015) also noted that AA patients demonstrated a higher prevalence of hypertension, diabetes mellitus, acute rejection, delayed graft function, and elevated incidence of cardiovascular diseases, when compared with non-AA patients. In contrast, in the present study, a significant difference between incidence of these diseases and the two ethnic groups examined was not detected (data not shown).

SNPs in genes involved in the phase I metabolism analyzed in this study were not markedly associated with graft rejection episodes. However, SNPs in phase II metabolism genes (UGT1A9 and UGT2B7) were associated with organ rejection episodes. After oral administration, the MMF prodrug is usually hydrolyzed to mycophenolic acid (MPA), its active metabolite. The immunosuppressive effect of MPA is inactivated by UGT1A9 enzyme through glucuronidation producing its major metabolite, the mycophenolic acid glucuronide (MPAG) (Picard et al., 2004). However, MPA is also inactivated by the UGT2B7 enzyme, generating mycophenolic acyl-glucuronide acid (AcMPAG) (Shipkova et al., 1999). This metabolite, although produced in lower amounts, may induce pro-inflammatory responses that subsequently lead to various adverse effects (Wieland et al., 2000).

In this study, it was observed that the association between genotypes (T/A and A/A) of SNPs rs6714486 (UGT1A9) enhanced the risk of graft rejection 1.6-fold. Our results corroborate those of Van Schaik et al. (2009), which also found a higher risk of rejection in transplant patients with the same allelic variants. The increased risk of rejection and its association with the UGT1A9 gene was explained by Girard et al. (2004), who demonstrated that the polymorphism rs6714486 was associated with a 1.4 -fold elevation in hepatic levels of the protein and a 1.9-fold increase in the glucuronidation of MPA. The enhanced expression and activity of UGT1A9 enzyme reduced the concentration of MPA and therefore diminished its immunosuppressive activity (Picard et al., 2004), promoting the initiation of organ rejection episodes.

Analysis of SNPs rs7662029 (UGT2B7 gene) showed that association of $A / G$ and A/A genotypes produced a 1.85-fold greater protection against graft rejection. The effect of this polymorphism on gene expression has not been elucidated. This polymorphism is in strong linkage disequilibrium (LD) with the polymorphism rs7438135. According to $\mathrm{Hu}$ et al. (2014), 23 SNPs mapped in the UGT2B7 promoter region are in LD. These authors also observed LD between SNPs rs7662029 and rs7438135 and reported that the haplotype carrying the $G$ allele for the rs7438135 showed a 50\% decrease in promoter activity of the gene and enzyme compared with the haplotype carrying the A allele (wild-type), thus identifying this polymorphism as functional. In the present study, the association of genotypes $A / G$ and $G / G$ demonstrated a non-significant trend toward protection, with an elevated 1.69-fold rise in protection against organ rejection episodes. This finding suggests that the presence of the $G$ allele may contribute to a lower activity of the enzyme and consequently reduced inactivation of MPA into AcMPAG, resulting in a lower risk of rejection.

As the rs7662029 polymorphism (genotype AA) is in LD with rs7438135 (genotype GG), it is possible that patients with a combination of these genotypes possess lower levels of enzymatic activity, eliminating immunosuppressive drugs more slowly. Univariate logistic regression analysis of the haplotypes formed between these two genotypes showed marked association with protection against rejection of almost 2 -fold. However, multivariate analysis, which considered the degree of HLA compatibility and ancestry, found no significant association (data not shown).

MPA metabolites (MPAG and AcMPAG) are eliminated through the bile by drug efflux transporter proteins ABCC2 and ABCG2 (Kobayashi et al., 2004; Miura et al., 2008). Subsequently, some metabolites undergo deconjugation by bacteria and are 
resorbed in the gastrointestinal tract through enterohepatic recirculation. This occurs in $10-60 \%$ of all MPAG, producing a second peak in MPA drug concentration (Bullingham et al., 1988). In this study, the association of $\mathrm{C} / \mathrm{A}$ and $\mathrm{A} / \mathrm{A}$ genotypes of SNP rs2231142 of the ABCG2 gene was associated with a 1.92 -fold increase in protection against organ rejection episodes. The presence of this SNP leads to an amino acid change, from lysine to glutamine, at codon 141, which decreases expression of the protein by $50 \%$ compared with the prevalent genotype (Tamura et al., 2007). Functionally, this SNP affects stability of the ABCG2 protein in the endoplasmic reticulum and raises susceptibility to ubiquitin-mediated degradation by the proteasome (Furukawa et al., 2009). Patients with the $\mathrm{C} / \mathrm{A}$ and A/A genotypes, compared with those with $\mathrm{C} / \mathrm{C}$ genotype, demonstrated an elevation in the concentration of plasmatic MPAG (Miura et al., 2008), possibly as a result of a reduced efflux of MPAG into the bile ducts. This may account for protective effect in carriers of the rs2231142 minor allele, because reduced expression of the ABCG2 transporter for rs2231142 is in agreement with findings of Miura et al., (2008). This study demonstrated for the first time the association between SNP rs2231142 and diminished risk of graft rejection. However, additional studies are required to clarify the effect of this SNP on pharmacokinetics of immunosuppressive MMF.

Of the 10 immune system genes analyzed in this study, only IL23 receptor (IL23R) showed association with graft rejection episodes. This gene is involved in the inflammatory process mediated by $\mathrm{T}$ helper 17 cells and plays an important role in autoimmune inflammation (Zhou et al., 2013). A study by Tsai et al. (2011) with 422 Chinese renal transplant patients showed that the $\mathrm{C}$ allele of the rs10889677 polymorphism leads to a 1.79fold increase in the risk of developing interstitial fibrosis and tubular atrophy in the graft. In the present study, the association of $\mathrm{A} / \mathrm{A}$ and $\mathrm{A} / \mathrm{C}$ genotypes of this SNP resulted in a 1.83-fold rise in graft rejection risk. Karimi et al. (2014) also observed an increase in the incidence of acute rejection in renal transplant male patients with the A/A genotype.

According Zheng et al. (2012) and Zhou et al. (2013), the presence of the polymorphic variant A of rs10889677 in the 3 '-UTR of the mRNA prevents binding of the microRNA let-7f and consequently increases transcription of IL23R. On the other hand, the $\mathrm{C}$ allele enhances the binding affinity of the microRNA let-7f and thus negatively regulates $I L 23 R$ expression (Zheng et al, 2012; Zhou et al., 2013). Vanden Eijnden et al. (2005) found elevated expression of IL23R, and amount of receptors present on the cell surface might assist in activation of IL23 interleukins, production of interferon- $\gamma$, and differentiation of T helper 1 cells, leading to increased inflammation and stimulation of pro-inflammatory cytokines levels. The findings cited above might account for the observed increased risk of rejection shown in our study.

In conclusion, this study demonstrated an association between SNPs rs7662029 (UGT2B7), rs6714486 (UGT1A9), rs2231142 (ABCG2), and rs10889677 (IL23R), and the incidence of graft rejection episodes in 246 Brazilian kidney transplant patients. Although transcription studies in cell lines to determine the functionality of SNPs were not conducted, these findings may contribute in the future to be used as candidate markers for screening kidney transplant patients with higher or lower rejection risk and thus help in medical management.

\section{Acknowledgments}

The authors thank the Kidney Institute of Londrina for support and partnership and the transplant patients that participated in the study. The authors are grateful for the assistance given by Ms. Sandra Lopes and Ms. Madalena Pikina (Clinilab, Londrina, Brazil).

\section{Declaration of interest}

The authors declare no conflicts of interest.

\section{Funding}

This study was supported by Conselho Nacional de Desenvolvimento Científico e Tecnológico (CNPq) [grant number 470398/2014-0] and Coordenação de Aperfeiçoamento de Pessoal de Nível Superior (CAPES/ PROAP). H.L. Cilião received scholarship of the Fundação Araucária de Apoio ao Desenvolvimento Científico e Tecnológico do Paraná. I.M.S. Cólus and S. R. Rogatto received investigator fellowship awards from $\mathrm{CNPq}$. 


\section{References}

Apel, H., Walschburger-Zorn, K., Häberle, L., Wach, S., Engehausen, D. G., and Wullich, B. 2013. De novo malignancies in renal transplant recipients: Experience at a single center with 1882 transplant patients over $39 \mathrm{yr}$. Clin. Transplant. 27: E30-E36.

Barrett, J. C., Fry, B., Maller, J., and Daly, M. J. 2005. Haploview: Analysis and visualization of LD and haplotype maps. Bioinformatics. 21: 263-265.

Bullingham, R. E., Nicholls, A. J., and Kamm, B. R. 1998. Clinical pharmacokinetics of mycophenolate mofetil. Clin. Pharmacokinet. 34: 429-455.

Burckart, G. J. and Amur, S. 2010. Update on the clinical pharmacogenomics of organ transplantation. Pharmacogenomics 11: 227-236.

Chapman, J. R., O’Connell, P. J., and Nankivell, B. J. 2005. Chronic renal allograft dysfunction. J. Am. Soc. Nephrol. 16: 3015-3026.

Elens, L., Bouamar, R., Hesselink, D. A., Haufroid, V., van der Heiden, I. P., van Gelder, T., and van Schaik, R. H. 2011. A new functional CYP3A4 intron 6 polymorphism significantly affects tacrolimus pharmacokinetics in kidney transplant recipients. Clin. Chem. 57: 1574-1583.

Fadili, W., Habib Allah, M., and Laouad, I. 2013. Chronic renal allograft dysfunction: Risk factors, immunology and prevention. Arab. J. Nephrol. Transplant. 6:45-50.

Furukawa, T., Wakabayashi, K., Tamura, A., Nakagawa, H., Morishima, Y., Osawa, Y., and Ishikawa, T. 2009. Major SNP (Q141K) variant of human ABC transporter ABCG2 undergoes lysosomal and proteasomal degradations. Pharm. Res. 26: 469-479.

Girard, H., Court, M. H., Bernard, O., Fortier, L. C., Villeneuve, L., Hao, Q., Greenblatt, D. J., von Moltke, L. L., Perussed, L., and Guillemette, C. 2004. Identification of common polymorphisms in the promoter of the UGT1A9 gene: evidence that UGT1A9 protein and activity levels are strongly genetically controlled in the liver. Pharmacogenetics. 14: 501-515.

Hebert, M. F. 1997. Contributions of hepatic and intestinal metabolism and P-glycoprotein to cyclosporine and tacrolimus oral drug delivery. Adv. Drug. Deliv. Rev. 27: 201-214.

Hesselink, D. A., van Schaik, R. H., van der Heiden, I. P., van der Werf, M., Gregoor, P. J., Lindemans, J., Weimar, W., and van Gelder, T. 2003. Genetic polymorphisms of the CYP3A4, CYP3A5, and MDR-1 genes and pharmacokinetics of the calcineurin inhibitors cyclosporine and tacrolimus. Clin. Pharmacol. Ther. 74: 245-254.

$\mathrm{Hu}$, D. G., Meech, R., Lu, L., McKinnon, R. A. and Mackenzie, P. I. 2014. Polymorphisms, and haplotypes of the UDP-glucuronosyltransferase 2B7 gene promoter. Drug. Metab. Dispos. 42: 854-862.

Karimi, M. H., Hejr, S., Geramizadeh, B., Yaghobi, R., Sagheb, M. M., and Kamali-Sarvestani, E. 2014. Combined analysis of cytokine gene polymorphism and the level of expression with allograft function in kidney transplant recipients. Transplant. Immunol. 30: 46-51.
Kobayashi, M., Saitoh, H., Kobayashi, M., Tadano, K., Takahashi, Y., and Hirano, T. 2004. Cyclosporin A, but not tacrolimus, inhibits the biliary excretion of mycophenolic acid glucuronide possibly mediated by multidrug resistance-associated protein 2 in rats. J. Pharmacol. Exp. Ther. 309: 1029-1035.

Miura, M., Kagaya, H., Satoh, S., Inoue, K., Saito, M., Habuchi, T., and Suzuki, T. 2008. Influence of drug transporters and UGT polymorphisms on pharmacokinetics of phenolic glucuronide metabolite of mycophenolic acid in Japanese renal transplant recipients. Ther. Drug. Monit. 30: 559-564.

MacPhee, I. A. M., Fredericks, S., Mohamed, M., Moreton, M., Carter, N. D., Johnston, A., Goldberg, L., and Holt, D. W. 2005. Tacrolimus pharmacogenetics: The CYP3A $5^{\star} 1$ allele predicts low dose-normalized tacrolimus blood concentrations in Whites and South Asians. Transplantation. 79: 499-502.

Ong, S. C. and Gaston, R. S. 2015. Medical management of chronic kidney disease in the renal transplant recipient. Curr. Opin. Nephrol. Hypertens. 24: 587-593.

Palanisamy, A. P., Schiltz, C. E., Pilch, N. A., Hunt, K. J., Nadig, S. N., Dowden, J. E., McGillicuddy, J. W., Baliga, P. K., Chavin, K. D., and Taber, D. J. 2015. Cardiovascular risk factors contribute to disparities in graft outcomes in African American renal transplant recipients: a retrospective analysis. Blood Press. 24: 14-22.

Picard, N., Ratanasavanh, D., Prémaud, A., Le Meur, Y., and Marquet, P. 2004. Identification of the UDP-glucuronosyltransferase isoforms involved in mycophenolic acid phase II metabolism. Drug. Metab. Dispos. 33: 139-146, 2004.

Pfaffl, M. 2001. A new mathematical model for relative quantification in real-time RT-PCR. Nucl. Acids. Res. 29: e45.

Ro, H., Min, S. I., Yang, J., Moon, K. C., Kim, Y. S., Kim, S. J., Ahn, C., and Ha, J. 2012. Impact of tacrolimus intraindividual variability and CYP3A5 genetic polymorphism on acute rejection in kidney transplantation. Ther. Drug. Monit. 34: 680-685.

Sattler, M., Guengerich, F. P., Yun, C. H., Christians, U., and Sewing, K. F. 1992. Cytochrome P-450 3A enzymes are responsible for biotransformation of FK506 and rapamycin in man and rat. Drug. Metab. Dispos. 20: 753-761.

Shipkova, M., Armstrong, V.W., Wieland, E., Niedmann, P. D., Schütz, E., Brenner-Weiss, G., Voihsel, M., Braun, F., and Oellerich, M. 1999. Identification of glucoside and carboxyl-linked glucuronide conjugates of mycophenolic acid in plasma of transplant recipients treated with mycophenolate mofetil. Br. J. Pharmacol. 126: 1075-1082.

Stephens, M., and Donnelly, P. 2003. A comparison of Bayesian methods for haplotype reconstruction from population genotype data. Am. J. Human. Genet. 73:1162-1169.

Tamura, A., Wakabayashi, K., Onishi, Y., Takeda, M., Ikegami, Y., Sawada, S., Tsuji, M., Matsuda, Y., and Ishikawa, T. 2007. Re-evaluation and functional classification of non-synonymous single nucleotide polymorphisms of the human ATP-binding cassette transporter ABCG2. Cancer. Sci. 98: 231-239. 
Tiercy, J. M. 2002. Molecular basis of HLA polymorphism: Implications in clinical transplantation. Transplant. Immunol. 9: 173-180.

Tsai, J. P., Yang, S. F., Wu, S. W., Hugn, T. W., Tsai, H C., Lian, J. D. and Chang, H. D. 2011. Association between interleukin 23 receptor polymorphism and kidney transplant outcomes: A 10-year Taiwan cohort study. Clin. Chim. Acta. 412: 958-962.

van Schaik, R. H., van Agteren, M., de Fijter, J. W., Hartmann, A., Schmidt, J., Budde, K., Kuypers, D., Le Meur, Y., van der Werf, M., Mamelok, R., and van Gelder, T. 2009. UGT1A9-275T>A/-2152C>T polymorphisms correlate with low MPA exposure and acute rejection in $\mathrm{MMF} /$ tacrolimus-treated kidney transplant patients. Clin. Pharmacol. Ther. 86: 319-327.

Vanden Eijnden, S., Goriely, S., De Wit, D., Willems, F., and Goldman, M. 2005. IL-23 up-regulates IL-10 and induces IL-17 synthesis by polyclonally activated naive $\mathrm{T}$ cells in human. Eur. J. Immunol. 35: 469-475.

Wieland, E., Shipkova, M., Schellhaas, U., Schütz, E., Niedmann, P. D., Armstrong, V. W., and Oellerich, M. 2000. Induction of cytokine release by the acyl glucuronide of mycophenolic acid: A link to side effects? Clin. Biochem. 33: 107-113.

Wolfe, R. A., Ashby, V. B., Milford, E. L., Ojo, A. O., Ettenger, R. E., Agodoa, L. Y., Held, P. J., and Port, F. K. 1999. Comparison of mortality in all patients on dialysis, patients on dialysis awaiting transplantation, and recipients of a first cadaveric transplant. N. Engl. J. Med. 341: 1725-1730.

Zheng, J., Jiang, L., Zhang, L., Yang, L., Deng, J., You, Y., Li, N., Wu, H., Li, W., Lu, J., and Zhou, Y. 2012. Functional genetic variations in the IL-23 receptor gene are associated with risk of breast, lung and nasopharyngeal cancer in Chinese populations. Carcinogenesis. 33: 2409-2016.

Zhou, S., Ruan, Y., Yu, H., Chen, Y., Yao, Y., Ma, Y., and Gao, Y. 2013. Functional IL-23R rs10889677 genetic polymorphism and risk of multiple solid tumors: A metaanalysis. PLoS One. 8: e80627.

Zununi Vahed, S., Ardalan, M., Samadi, N., and Omidi, Y. 2015. Pharmacogenetics and drug-induced nephrotoxicity in renal transplant recipients. Bioimpacts. 5: 45-54. 